\title{
Dynamic Compensation of Torsional Oscillation in Paper Machine Sections
}

\author{
M. Aníbal Valenzuela, Senior Member, IEEE, John Martin Bentley, Life Fellow, IEEE, \\ Andrés Villablanca, and Robert D. Lorenz, Fellow, IEEE
}

\begin{abstract}
Torsional oscillations bound the velocity loop bandwidth (BW) of industrial drives and servo systems, deteriorating the transient response to commands and disturbances. This is especially harmful in multidrive systems like paper machines that need to run synchronized. This paper presents two methods to dynamically compensate the torsional oscillations produced by the compliant components between the motor and the load: either a notch filter or an acceleration feedback based on the rigid-body Luenberger observer. The evaluation includes the effect of reducer backlash, whereby both time and frequency domain responses are used to compare the benefits of each technique in a typical paper machine section. Results show that both methods, if properly tuned, are effective techniques to reduce torsional oscillations and are robust to parameter variations.
\end{abstract}

Index Terms-Backlash, frequency response analysis, resonance, torsional oscillations, torsional stiffness, two mass modeling.

\section{NOMENCLATURE}

$G_{\text {notch }}$

$I_{a}$

$J_{m}, J_{L}$

Transfer function of notch filter.

Motor armature current.

Motor (and reducer) and load (rolls)

inertias.

$J_{r} \quad$ Total inertia reflected to the motor axis.

$K_{s h}$ Torsional stiffness of shaft.

$J_{a}$

$K_{c}$

$K_{e}, K_{t}$

$K_{p \_i}, K_{p \_v}$

Active inertia gain.

Converter voltage gain.

Motor back EMF and torque constants.

Proportional gains of current and velocity controllers.

$K_{p \_o}, K_{i \_o}, K_{d \_o}$ Controller gains for the rigid-body observer.

$R_{a}, L_{a}$

Paper PID-05-08, presented at the 2005 IEEE Pulp and Paper Industry Conference, Jacksonville, FL, June 19-23, and approved for publication in the IEEE TRANSACTIONS ON Industry APPLICATIONS by the Pulp and Paper Industry Committee of the IEEE Industry Applications Society. Manuscript submitted for review June 23, 2005 and released for publication September 1, 2005. This work was supported by CONICYT-Chile through the Fondecyt Project No. 1050945 and by the Wisconsin Electric Machines and Power Electronics Consortium of the University of Wisconsin-Madison.

M. A. Valenzuela and A. Villablanca are with the Department of Electrical Engineering, University of Concepción, Concepción, Chile (e-mail: anivalenz@udec.cl; anvillab@udec.cl).

J. M. Bentley is at 6239 Clearview Drive, Manitowish Waters, WI 54545 USA (e-mail: jmbentley@centurytel.net).

R. D. Lorenz is with the Mead Witter Foundation, Wisconsin Rapids, WI 54495 USA, and also with the Departments of Mechanical Engineering and Electronics and Communications Engineering, University of Wisconsin at Madison, Madison, WI 53706-1572 USA (e-mail: lorenz@engr.wisc.edu).

Digital Object Identifier 10.1109/TIA.2005.858317

TABLE I

SECTION CHARACTERISTICS

\begin{tabular}{|c|c|c|c|c|}
\hline \multirow{3}{*}{ SECTION } & \multicolumn{2}{|c|}{ TAPPI Power } & \multirow{2}{*}{$\begin{array}{c}\text { Moment of Inertia } \\
\text { (@ roll) }\end{array}$} & \multirow{3}{*}{$\begin{array}{l}\text { Reducer } \\
\text { Gear Ratic }\end{array}$} \\
\hline & NRL & $\mathrm{RDC}$ & & \\
\hline & {$[\mathrm{KW}]$} & [KW] & {$\left[\mathrm{Kg}-\mathrm{m}^{2}\right]$} & \\
\hline - Size Press & 94.6 & 123.0 & 1057 & 6.2 \\
\hline - Dryer & 83.2 & 179.2 & 76005 & $2 * 6.2$ \\
\hline - Calender & 85.3 & 113.5 & 1961 & 5.06 \\
\hline
\end{tabular}

TABLE II

RESONANT FREQUENCIES AND MAGNITUdES

\begin{tabular}{lcccc}
\hline \hline \multirow{2}{*}{ SECTION } & \multicolumn{2}{c}{ Anti-resonance } & \multicolumn{2}{c}{ Resonance } \\
\cline { 2 - 5 } & $\mathrm{f}_{\mathrm{ar}}[\mathrm{Hz}]$ & Mag_f $_{\mathrm{ar}}[\mathrm{db}]$ & $\mathrm{f}_{\mathrm{r}}[\mathrm{Hz}]$ & ${\text { Mag_ } \mathrm{f}_{\mathrm{r}}[\mathrm{db}]}$ \\
\hline - Size Press & 1.60 & -112.7 & 6.32 & 20.99 \\
- Dryer & 0.45 & -136.7 & 5.59 & 17.22 \\
- Calender & 2.00 & -138.6 & 16.60 & 25.54 \\
\hline
\end{tabular}

$\omega^{*}$

$T_{L}, T_{s h}$

$T_{i}, T_{v}$

$b_{s h}$

$b_{L}, b_{m}$

$\hat{\theta}_{m}, \hat{\omega}_{m}, \hat{\alpha}_{m}$

$\delta$

$\zeta_{z}, \zeta_{p}$

$\theta_{L}, \theta_{m}, \omega_{L}, \omega_{m}$

$\omega_{n}$

$\hat{\omega_{r}}$

$\hat{X}$
Velocity command reference.

Load and shaft torques.

Integral times of current and velocity controllers.

Damping factor of shaft.

Load and motor damping factors.

Estimated position, velocity, and acceleration.

Backlash angle between pinion and gear (reducer).

Zero and pole damping coefficients of notch filter.

Load and motor positions and velocities.

Notch frequency.

Resonant frequency.

Estimated values display $\wedge$ overstrike.

\section{INTRODUCTION}

Torsional oscillations are one of the most significant issues that bound the velocity loop bandwidth (BW) of industrial drives and servo systems. In a paper machine, where all the mechanical sections must run in a coordinated or synchronized fashion, the sections that have the lowest resonant frequencies will determine the transient response of the whole machine.

In [1], the authors evaluated torsional oscillations in typical sections of a paper machine and explored different options to mitigate these oscillations and/or to increase the resonant frequency. The options included designing stiffer mechanical 


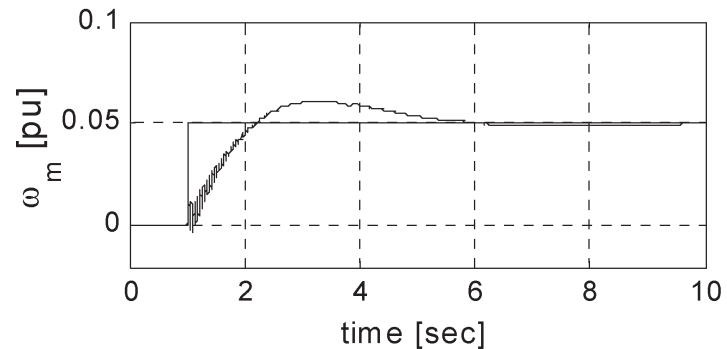

(a)

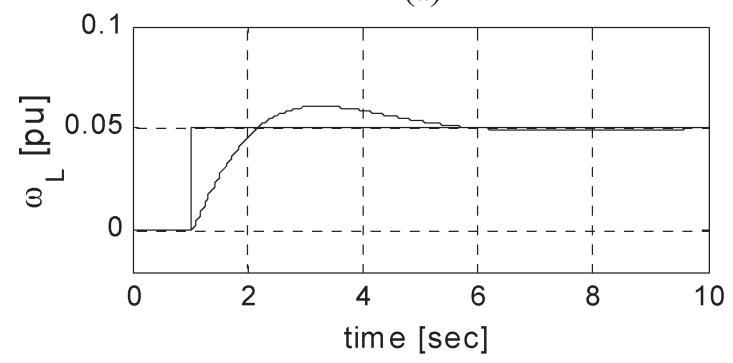

(b)

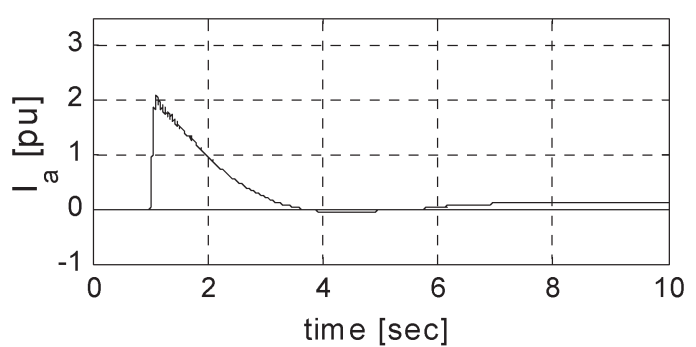

(c)

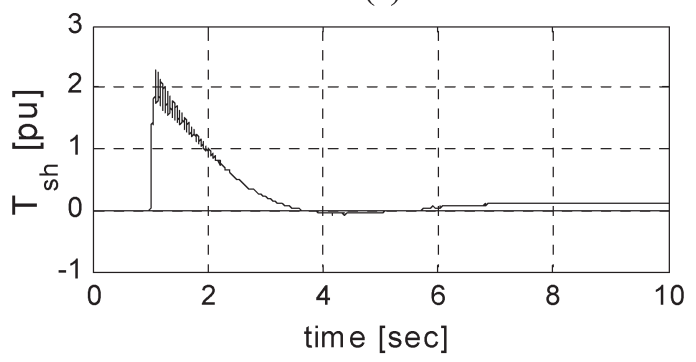

(d)

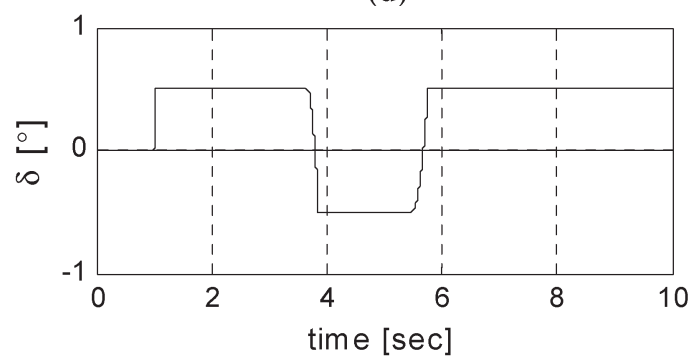

(e)

Fig. 1. Time response of Calender section to a 5\% step command. (a) Motor velocity. (b) Rolls velocity. (c) Motor current. (d) Shaft torque. (e) Reducer angle.

drive trains using shafts with larger diameters and shorter lengths, the use of hollow shafts, and tuning the velocity loops for overdamped response. Although these techniques help to achieve better and faster response, there is still a need for further improvements, especially in sections with higher load inertias, which have low resonant frequencies.

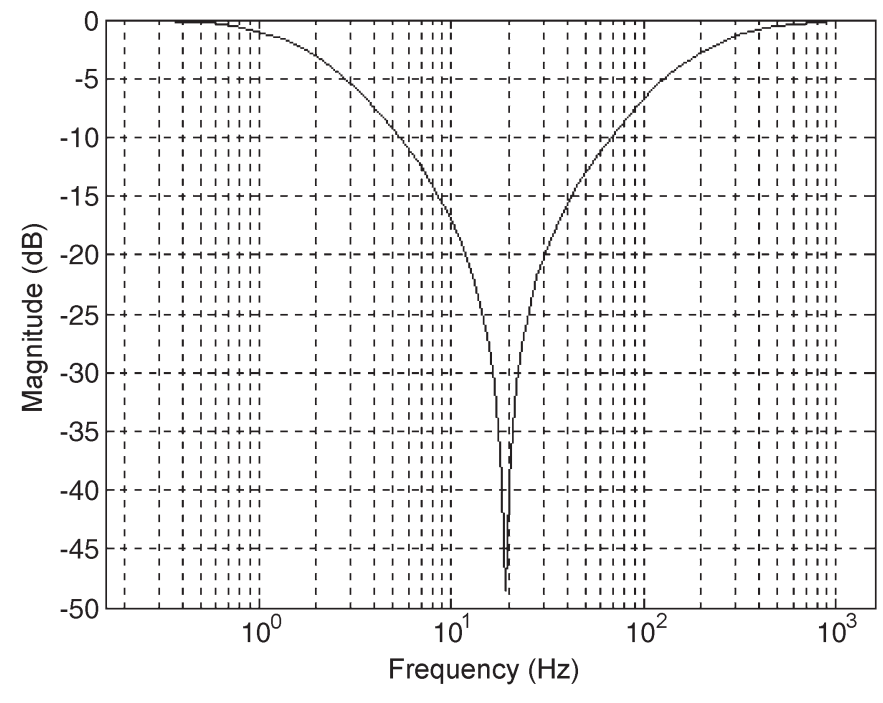

Fig. 2. Frequency response of the pole/zero cancellation notch filter.

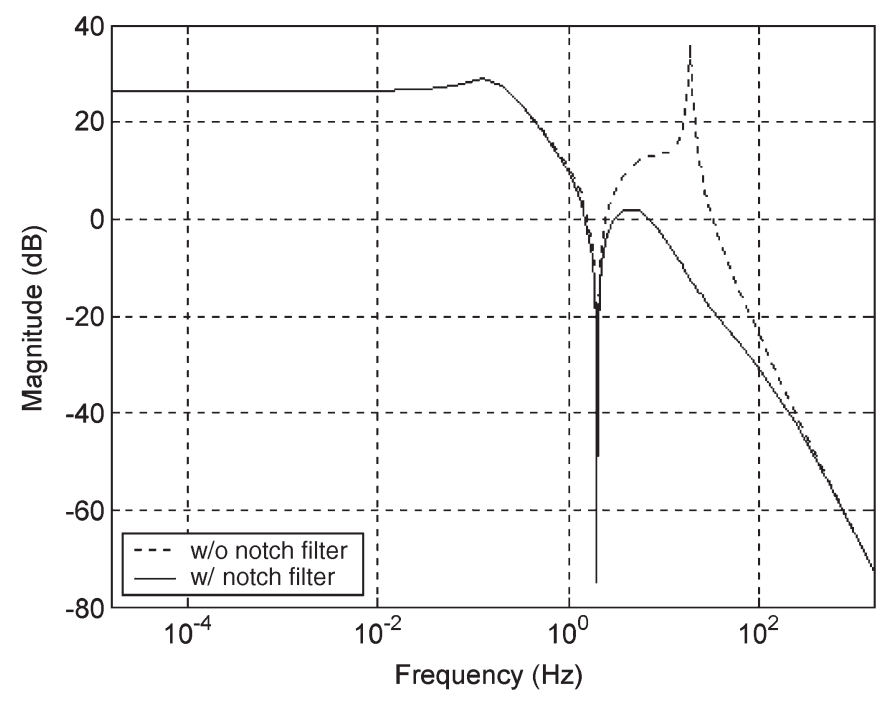

Fig. 3. Closed-loop frequency response of Calender section with and without the notch filter (1).

Several papers have been reported proposing and evaluating different methods of controlling or reducing torsional oscillations [2]-[8]. Most of them are applied to high-performance servo systems with resonant frequency in the range of 100-200 Hz. A representative paper of the different compensating techniques proposed is [5], where the authors compared seven methods, including low-pass, notch, and bi-quad filtering methods, and observer-based methods using rigid-body and compliant-body observers. Evaluations were done in a servo system with a $100-\mathrm{Hz}$ resonant frequency. Best results were obtained using notch filter and acceleration feedback based on the rigid-body Luenberger observer. In [6], acceleration feedback was evaluated for a system with a relatively low resonant frequency. The results demonstrated a significant improvement in dynamic performance.

The application of any of these compensation techniques to paper machine sections needs to consider real-life operating conditions that affect both field tuning of the power trains and long-term stability. The two main considerations are as 


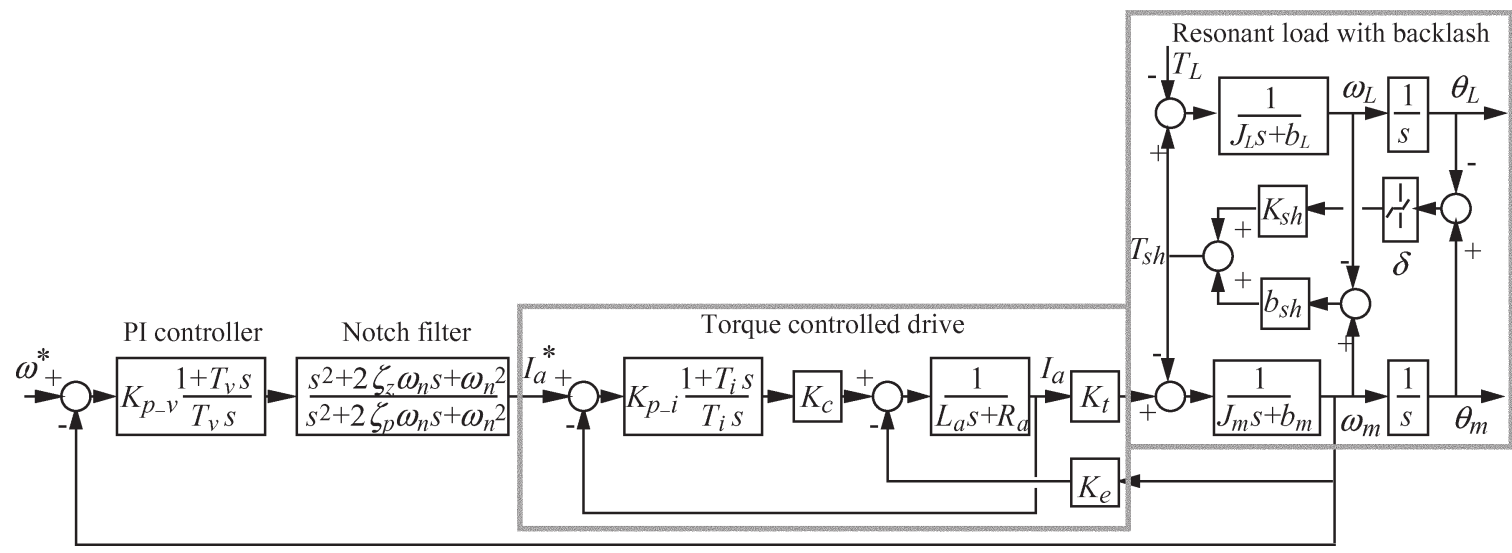

Fig. 4. Block diagram of the resonant system motor velocity loop including a notch filter.

follows. 1) The physical damping factor in most indrives depends on several machine parameters, such as fluid viscosity and plasticity of roll covers. A change in the damping factor produces a change in the resonant frequency, which affects the performance of filter-based methods. 2) Mechanical sections with backlash operating at low torque, such as a dryer section unloaded to almost zero indrive torque due to the outgoing sheet tension, present a situation where the load inertia appears to be disconnected momentarily from the motor. Depending on the compensating technique, this condition could move the system to an operating region where backlash-induced oscillation occurs.

This paper addresses the detailed evaluation of two compensating techniques applied to the dry-end section of an light weight coated (LWC) paper machine [1]. The evaluation considers the effect of reducer backlash, and special attention is given to the tuning criteria for the compensator parameters and to the robustness of the compensating techniques to physical parameter variations.

\section{B ASELINE SySTEM}

The dry-end sections of the LWC paper machine reported in [1] are used as the baseline system for the evaluation of alternative compensation techniques. Table I shows the main characteristics of each section and Table II summarizes the resonant and anti-resonant frequencies.

The baseline system velocity loop tuning for each section with specified load inertias and jackshafts is for $15 \%$ overshot and 0.707 damping factor. This tuning criterion will allow triggering of torsional oscillation due to backlash during torque reversals.

Fig. 1 shows the time-domain response of the Calender section to a 5\% step command, with the velocity controller gains set as stated. Torque switches sign at $t=3.5 \mathrm{~s}$ and $\mathrm{t}=5.6 \mathrm{~s}$ [see Fig. 1(d)], which results in the load inertia being momentarily disconnected, while the reducer angle $\delta$ moves from $+0.5^{\circ}$ to $-0.5^{\circ}$ or vice versa [Fig. 1(e)]. Right after the step command and after each torque reversal, torsional oscillation appears. By zooming in on that oscillation, it can be seen that the dominant frequency is $19.1 \mathrm{~Hz}$. From Table II, the physical resonant frequency of the system is $16.6 \mathrm{~Hz}$. This difference can be explained by the movement of the system poles after closing the feedback loop. This effect can be confirmed by drawing the root locus for the entire system (using Matlab or equivalent software).

This system will serve as the baseline. The benefits of each of the dynamic compensation techniques will be evaluated by comparing both the frequency and the time-domain response of the compensated system to the equivalent response of the baseline system without compensation.

\section{NotCh FILTER COMPENSATION}

The transfer function of a two-inertia system coupled by a compliant shaft and/or gearbox shows that there are complex poles near the imaginary axis that represent the lightly damped torsional resonance typical of such mechanical systems. After closing the velocity loop, these poles move slightly and become somewhat better damped.

One of the well-established techniques to attenuate the effect of these resonant poles is to introduce a series filter with a transfer function comprised of a set of complex zeros that theoretically cancel the resonant poles of the plant, and a set of well damped poles that are located in a more stable (well damped) position in the left half plane (LHP). If implemented precisely, this pole/zero compensation would theoretically produce a desirable well-damped dynamic response characteristic.

One possible transfer function for this notch filter is given by

$$
G_{\mathrm{notch}}(s)=\frac{s^{2}+2 \zeta_{z} \omega_{n} s+\omega_{n}^{2}}{s^{2}+2 \zeta_{p} \omega_{n} s+\omega_{n}^{2}}
$$

This transfer function is composed of two zeros located in the LHP, but very close to the imaginary axis, and two well-damped poles at the same frequency. The two zeros are theoretically tuned to cancel the resonant poles of the system.

Fig. 2 shows the magnitude frequency response of the notch filter. As expected, it produces significant attenuation at notch frequency without affecting the system response at lower and higher frequencies.

\section{A. Notch Filter Tuning}

In the frequency domain, tuning of the notch filter (1) requires cancellation of the closed-loop complex poles of the 


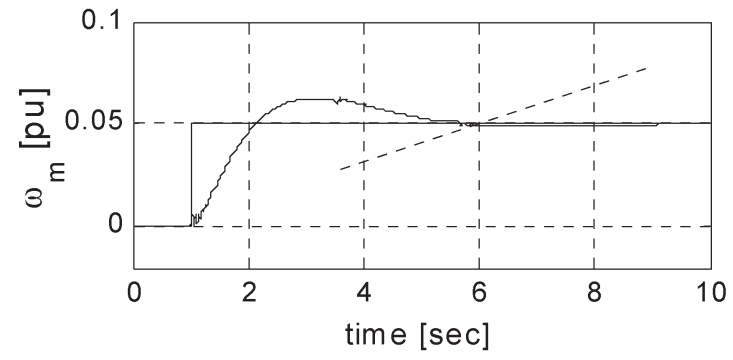

(a)

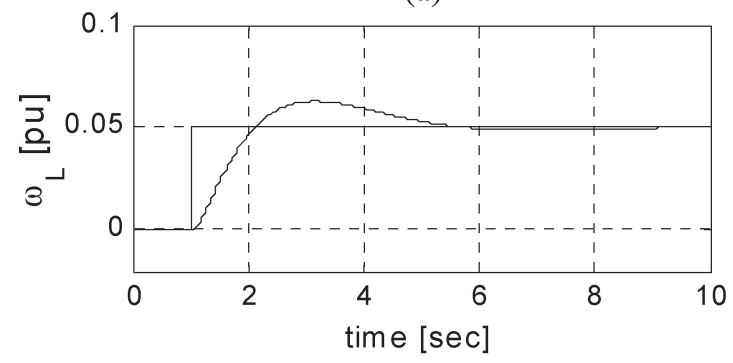

(b)

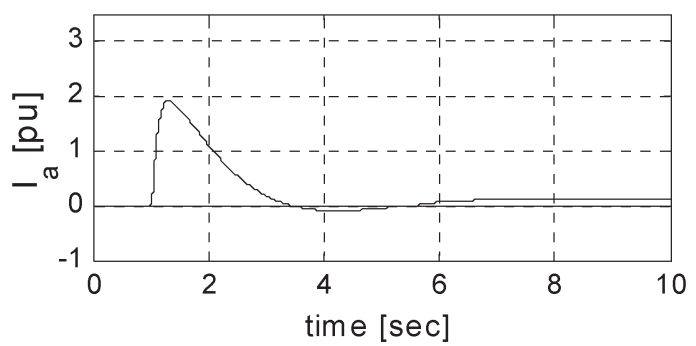

(c)

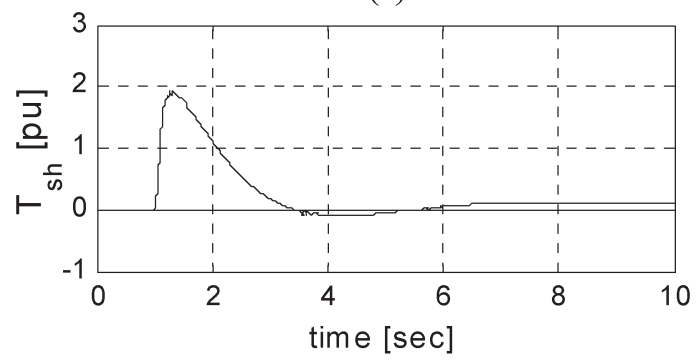

(d)

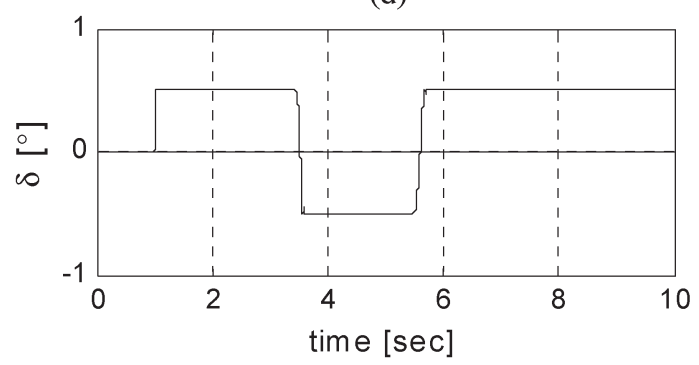

(e)

Fig. 5. Time response of the Calender section to a 5\% step command including notch filter. (a) Motor velocity. (b) Load (Rolls) velocity. (c) Motor current. (d) Shaft torque. (e) Reducer angle.

resonant system and selecting the appropriate attenuation (desired damping factor) at resonant frequency.

The steps that should be followed are as follows. 1) Obtain the closed-loop poles of the resonant system, i.e., including the effect of velocity feedback, and set the zeros of the notch filter to cancel these resonant poles. 2) From the velocity loop

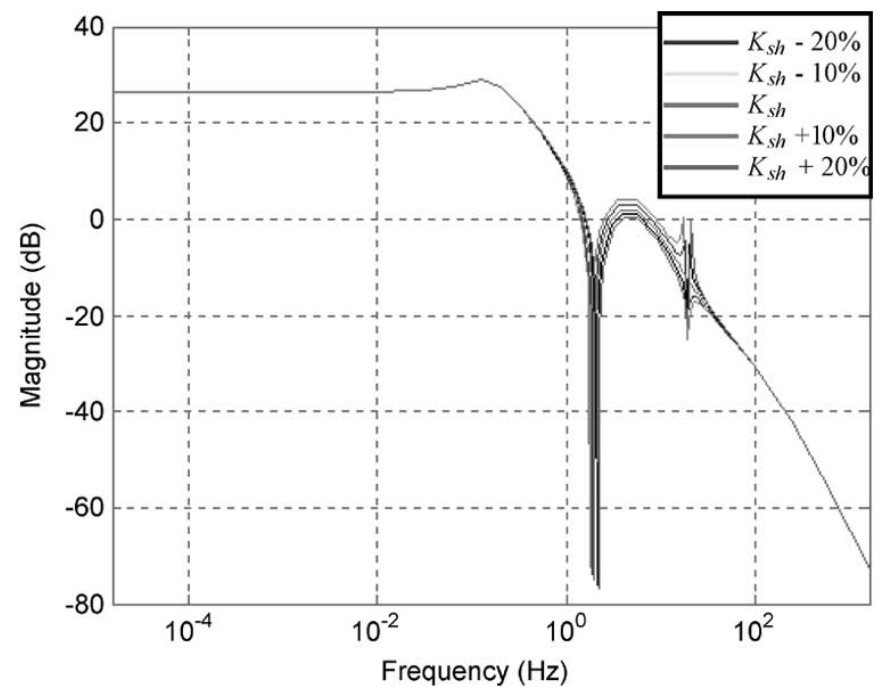

Fig. 6. Effect of variations in the shaft torsional stiffness $K_{s h}$ on the closedloop frequency response when using a notch filter.

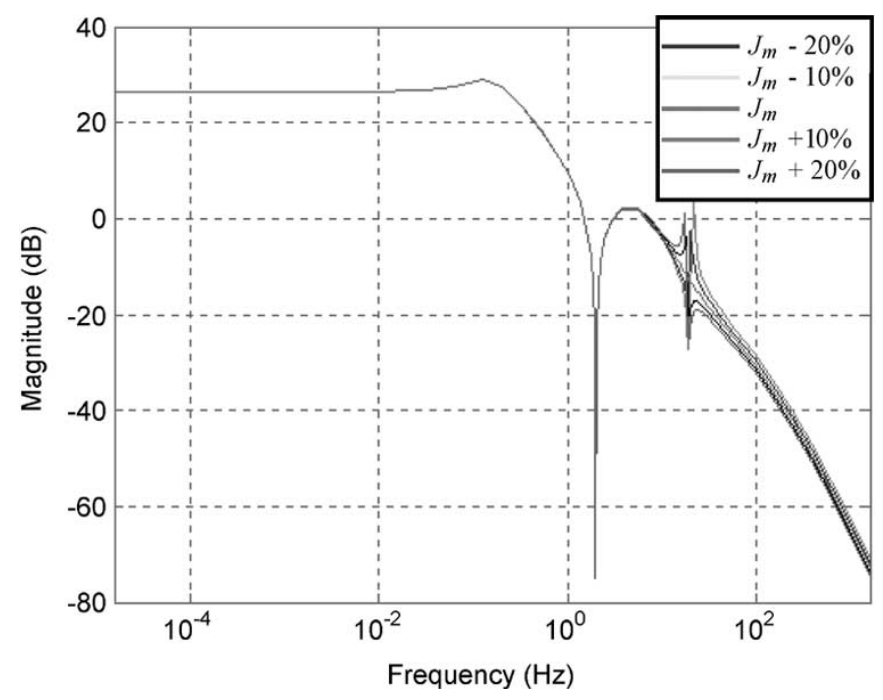

Fig. 7. Effect of variations in the motor inertia $J_{m}$ on the closed-loop frequency response when using a notch filter.

frequency response plot, determine the attenuation required to compensate the high magnitude peak at resonant frequency.

Evaluating the transfer function of the notch filter shows that the attenuation at resonant frequency is a function of the ratio of the actual system damping factor $\zeta_{z}$ to the desired system damping factor $\zeta_{p}$, i.e.,

$$
\left|G_{\text {notch }}\left(j \omega_{r}\right)\right|=\frac{\zeta_{z}}{\zeta_{p}} .
$$

Therefore, knowing the attenuation required will determine the desired damping ratio (2) and complete the theoretical tuning procedure.

Applying these steps to the Calender section, it was found that the closed-loop resonant poles are located at $(-2.1 \pm$ $120 \mathrm{j}$ ) and the resonant frequency is $19.1 \mathrm{~Hz}$. From Fig. 3, the velocity loop frequency response of the resonant system 


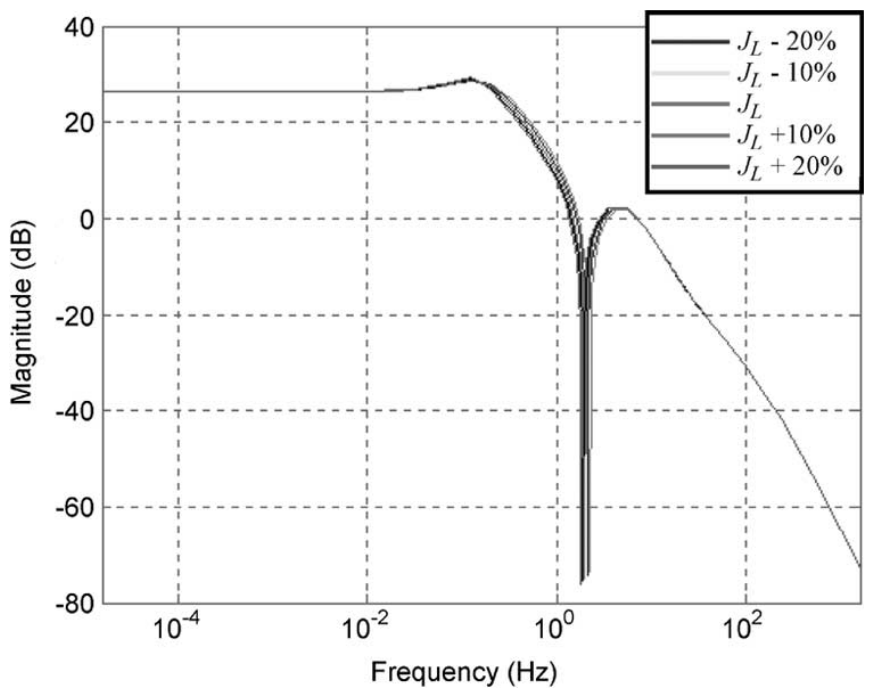

Fig. 8. Effect of variations of rolls inertia $J_{L}$ on the closed-loop frequency response when using a notch filter.

and the desired compensated frequency response, the required attenuation can be found to be $-48.5 \mathrm{~dB}$.

With these values, the notch filter transfer function becomes

$$
G_{\text {notch }}(s)=\frac{s^{2}+4.4 s+14400}{s^{2}+1168 s+14400} .
$$

\section{B. Time Response}

Fig. 4 shows the block diagram of the resonant load drive system, including backlash, current and velocity loops, and the notch filter. Using this model, the time-domain response of the Calender section to a 5\% step command is obtained.

Fig. 5 shows the time-domain response of the motor and load (roll) velocities, motor current, shaft torque, and the delta angle between the pinion and the gear in the reducer.

The comparison of these results to the response of the Calender section without the notch filter in Fig. 1 clearly demonstrates the benefits of the notch filter achieving ideal pole/zero compensation. The magnitude of the oscillation is greatly reduced and the oscillation is rapidly damped out.

\section{Robustness to Parameter Variations}

Although the compensation of torsional oscillations using the notch filter has proven to be very effective, it is necessary to evaluate the robustness of the compensation technique to physical parameter variations. These variations may be produced by changes in the parameters due to the operating conditions or due to errors in the measurement or estimation of these parameters. In the first case, the resonant frequency varies from the one used for the notch filter's pole zero cancellation; and in the second case, the computed resonant frequency simply does not match the actual value. Both cases result in detuned pole/zero compensation.

The effect of such mistuning can be evaluated using the closed-loop velocity response. In this case, the evaluation considered $\pm 10 \%$ and $\pm 20 \%$ changes in the main parameters that

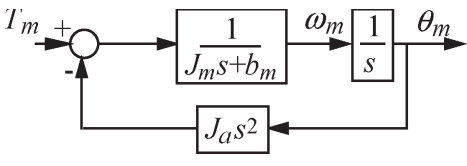

Fig. 9. Simplified block diagram for acceleration feedback.

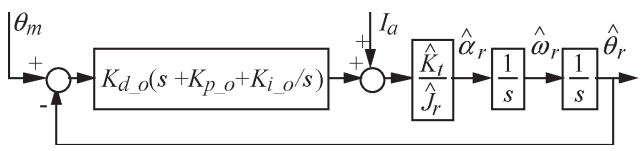

Fig. 10. Rigid-body Luenberger-style observer for the motor with a rigidly connected load [5].

affect the resonant and anti-resonant frequencies. The parameters considered are the shaft torsional stiffness $K_{s h}$, the motor (and reducer) inertia $J_{m}$, and the load (rolls) inertia $J_{L}$. Results are shown in Figs. 6, 7, and 8, respectively.

According to Figs. 6 and 7, variations in $K_{s h}$ and $J_{m}$ produce a small shift of the resonant frequency. As the notch filter maintains its location, there is a partial detuning of the compensating effect that shows up in the frequency response as a secondary maximum/minimum pair, but with magnitudes that are not problematic for stability. For this case, the benefits of the notch filter compensation are still significant for $\pm 20 \%$ changes in $K_{s h}$ or $J_{m}$.

Fig. 8 shows that changes in $J_{L}$ have negligible effects on compensation. This is consistent with the fact that the resonant frequency is almost independent of $J_{L}$ when $J_{L}$ is much greater than $J_{m}$.

Based on these results, it can be concluded that notch filter compensation is relatively robust to changes in the resonant system parameters of the magnitude investigated in this work.

\section{OBSERVER-BASED ACCELERATION FEEDBACK COMPENSATION}

\section{A. Acceleration Feedback}

Acceleration feedback is an effective method of reducing drive sensitivity to mechanical resonance [5]-[8]. Its effect can be evaluated from the block diagram in Fig. 9.

Solving for the motor velocity transfer function based on this simplified model results in

$$
\frac{s \theta_{m}}{T_{m}}=\frac{1}{\left(J_{m}+J_{a}\right) s+b_{m}} .
$$

Therefore, the effect of acceleration feedback is often described as an equivalent increase in drive inertia to $\left(J_{m}+J_{a}\right)$, whereby $J_{a}$ can be thought of as an active inertia, measured in kilogram per square meter units [7]. This active inertia is superior because it does not require adding extra flywheels to increase the physical inertia and does not limit the response of the drive to fast commands as adding physical inertia would [7]. 


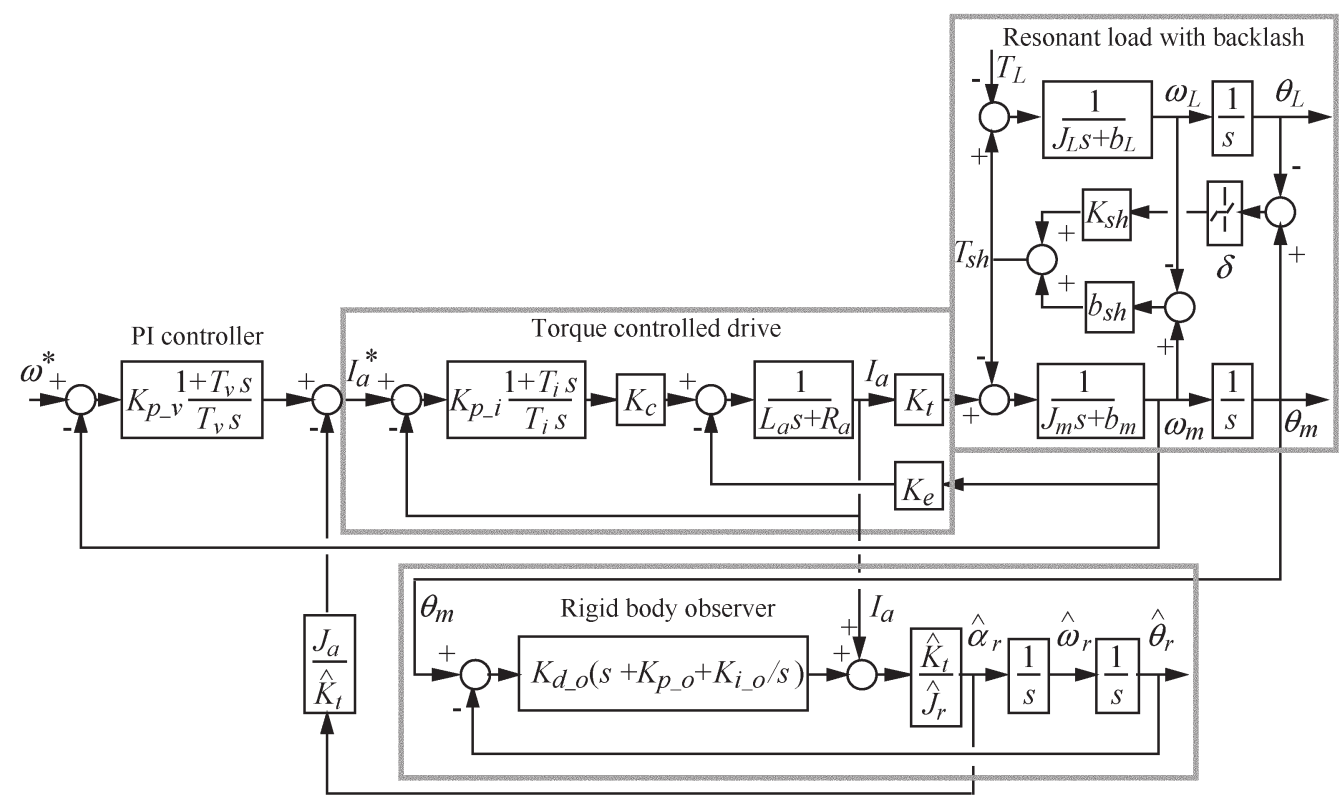

Fig. 11. Block diagram of the resonant system motor velocity loop including acceleration feedback compensation using a Luenberger observer.

\section{B. Rigid-Body Luenberger Observer}

In order to implement acceleration feedback, there is a need for an acceleration feedback signal. Double differentiation of the position signal is not appropriate because the acceleration signal that results is badly contaminated with quantization noise and has an inherent lagging property, even without filtering. Therefore, a better option is to use an observer [7]-[9].

The proposed observer is the rigid-body Luenberger-style observer [5] represented by the block diagram shown in Fig. 10. The observer structure is analogous to the motor model with the load inertia connected by a rigid coupling, i.e., $J_{r}=J_{m}+J_{L}$. The required inputs are the measured motor position $\theta_{m}$ and the measured (or commanded) motor armature current $I_{a}$. The measured position is used for feedback control to force the observer to track the actual position. The motor current is used as a feed-forward signal to the observer. For feedback control, the estimated position $\hat{\theta}_{m}$ is compared to the actual position $\theta_{m}$ and the error is inputted to a proportional, integral, and derivative (PID) controller used to drive the steady-state estimation error to zero. The output of the compensator is added to the feedforward motor current signal, and the resulting signal is applied to the motor block model, which produces the estimated acceleration $\hat{\alpha}_{m}$, estimated velocity $\hat{\omega}_{m}$, and estimated position $\hat{\theta}_{m}$.

Developing the transfer function of the rigid-body observer and plotting the $\hat{\theta}_{m} / \theta_{m}$ estimation accuracy frequency response with respect to the measured position signal, it can be seen that the Luenberger observer behaves like a low-pass filter for the unmodeled resonant part of the position signal. If the observer $\mathrm{BW}$ is lower than the structural resonance, then the low-pass filter properties of the observer will attenuate the resonant frequency components of the position signal, which helps to reduce the resonant oscillation.

There are different options to tune the observer's PID controller. For this evaluation, the tuning was selected to make the observer filter dynamics equivalent to a three-pole Butterworth filter, which gives a flat frequency response in the filter BW. A third-order Butterworth filter has the characteristic expression $\left(s+\omega_{r}\right)\left(s^{2}+\omega_{r} s+\omega_{r}^{2}\right)$, which gives

$$
\begin{aligned}
& K_{p_{-_{o}}}=\omega_{r} \\
& K_{i_{-o}}=\frac{\omega_{r}^{2}}{2} \\
& K_{d \__{o}}=\frac{\hat{J}_{m}}{\hat{K}_{T}} 2 \omega_{r}
\end{aligned}
$$

for the observer gains (whereby $\omega_{r}$ was selected to be $120 \mathrm{rad} / \mathrm{s}$ ).

\section{Acceleration Feedback Compensation}

Fig. 11 shows the complete block diagram of the resonant system motor velocity loop including acceleration feedback compensation implemented using the rigid-body Luenberger observer. The acceleration feedback is subtracted from the current reference, and therefore, the estimated acceleration feedback is scaled to give current units.

The effects of acceleration feedback compensation can be interpreted from the closed-loop frequency response plots of the system with and without compensation as shown in Fig. 12.

It can be seen that the resonant frequency has moved to a new location and its magnitude is greatly reduced. Specifically, for the Calender section, the resonant frequency is now $2.75 \mathrm{~Hz}$ and its magnitude is around $10 \mathrm{~dB}$. This new value falls inside the velocity controller BW, and therefore the velocity controller will respond trying to keep the actual velocity close to its reference. 


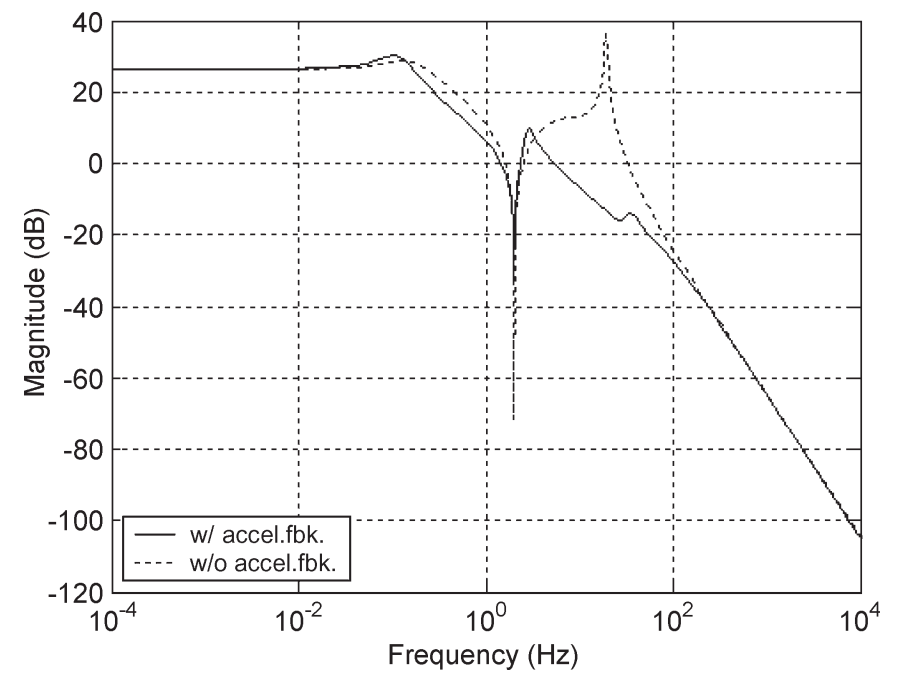

Fig. 12. Closed-loop frequency response of the Calender section with and without observer-based acceleration feedback compensation.

\section{Time Response}

To facilitate the comparison between acceleration feedback and notch filter compensation methods, the same 5\% step command was applied to the Calender section and the same variables shown in Fig. 5(a) and (b) were plotted. The resulting waveforms can be seen in Fig. 13(a) and (b).

Looking at the motor and load velocity waveforms, they are almost free of oscillation and follow the step command according to the dynamic response set for the velocity controller. In contrast, the armature current and shaft torque show a lowfrequency moderately well damped oscillation superimposed on their response to the step commands. The frequency of these oscillations is $2.75 \mathrm{~Hz}$, which corresponds to the new location of the resonant frequency, as it can be seen in the frequency response plot of Fig. 12. For the compensated system, the new resonant frequency falls inside the velocity controller BW, and therefore the velocity loop commands an oscillatory current reference to dampen oscillation in the velocity response. As the magnitude of the resonant frequency of the compensated system is moderate (about $10 \mathrm{~dB}$ for the Calender section), the peak current, although high, is considered acceptable (for a theoretical 5\% step command, it reaches 2.7 p.u. in the first period of oscillation).

Based on these results, it can be concluded that acceleration feedback compensation based on a rigid-body Luenberger observer is an effective technique to reduce torsional oscillation in resonant drive systems.

\section{E. Robustness to Parameter Variations}

Consistent with the robustness evaluation done for notch filter compensation, the robustness of acceleration feedback compensation to parameter variations was evaluated by plotting the frequency response assuming $\pm 10 \%$ and $\pm 20 \%$ variations in the shaft torsional stiffness $K_{s h}$, in the motor inertia $J_{m}$, and in the load (rolls) inertia $J_{L}$. Results are shown in Figs. 14, 15, and 16 , respectively.

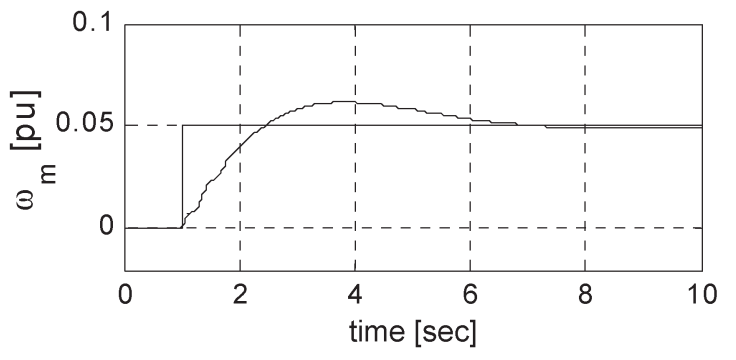

(a)

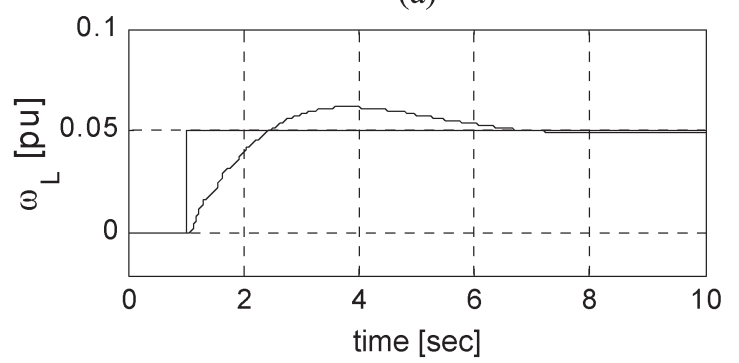

(b)

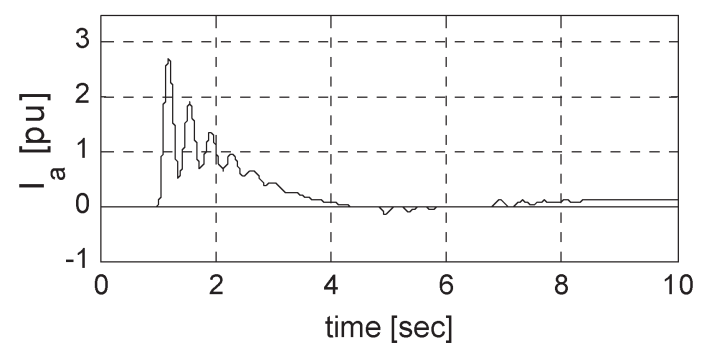

(c)

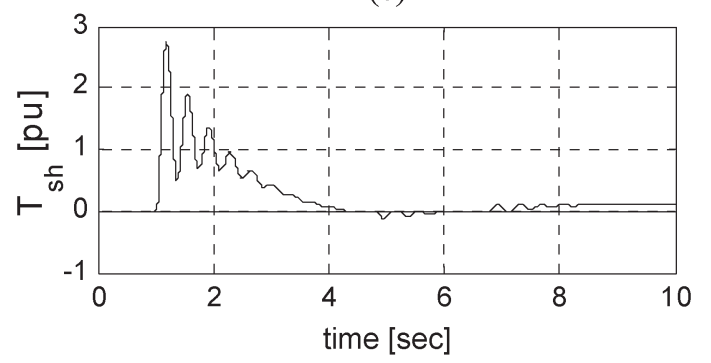

(d)

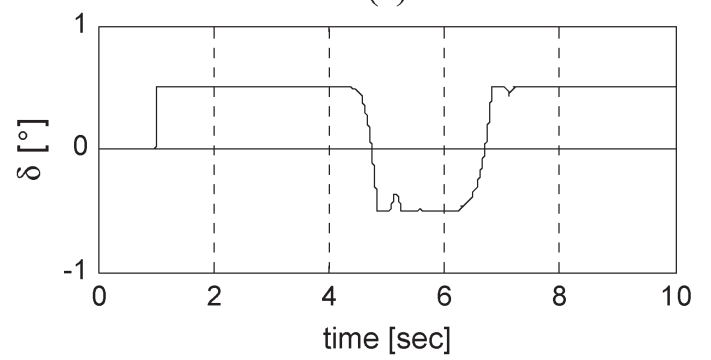

(e)

Fig. 13. Time response of the Calender section to a 5\% step command including acceleration feedback compensation. (a) Motor velocity. (b) Rolls velocity. (c) Motor armature current. (d) Shaft torque. (e) Reducer angle.

The conclusions that can be drawn from these plots are similar to those for the notch filter. A $\pm 20 \%$ variation in the main parameters of the drive only produces minor variations in the frequency response plots (and therefore, in time response). From Figs. 14 and 16, variations in torsional stiffness $K_{s h}$ and 


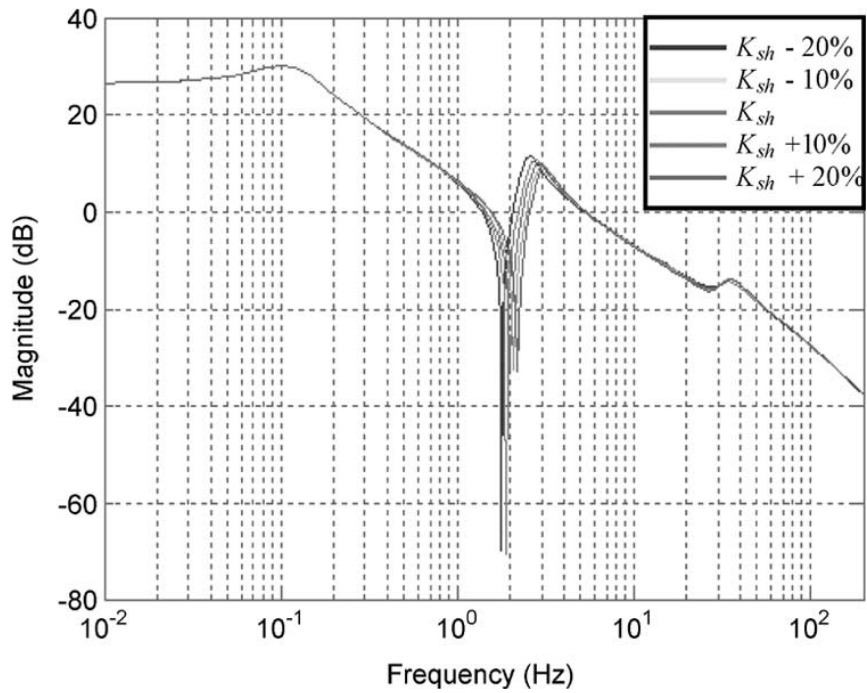

Fig. 14. Effect of variations of the shaft torsional stiffness $K_{s h}$ on the closedloop frequency response when using observer-based acceleration feedback.

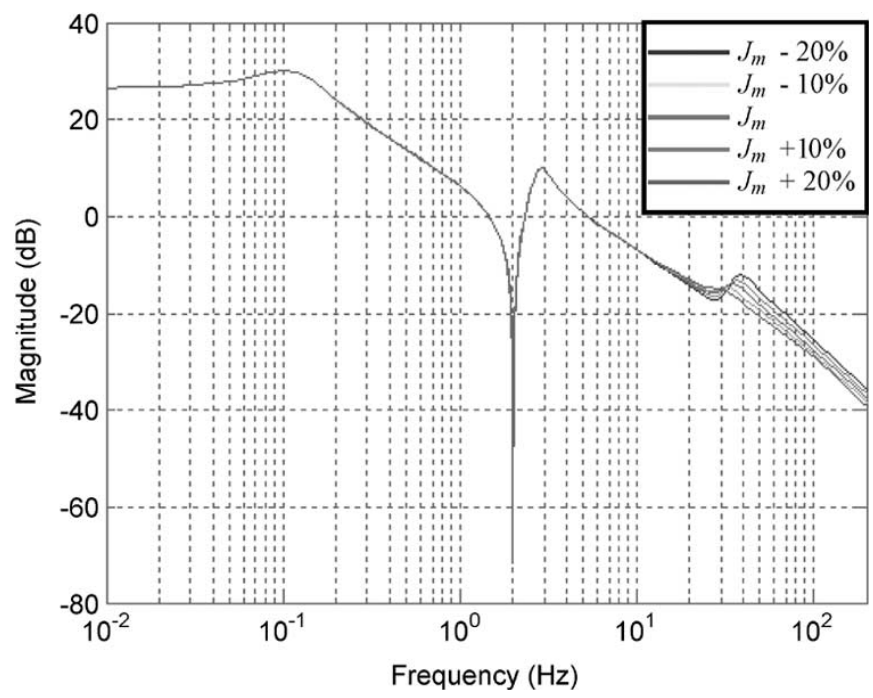

Fig. 15. Effect of variations of motor inertia $J_{m}$ on the closed-loop frequency response when using observer-based acceleration feedback.

load (rolls) inertia $J_{L}$ have moderate effects on the resonant frequency location and magnitude that will produce moderate increases in the current (and torque) oscillation. Instead, variations in $J_{m}$ do not change the resonant frequency.

Based on these results, it can be concluded that acceleration feedback compensation is relatively robust to changes in the resonant system parameters of the magnitude investigated in this paper.

\section{Conclusion}

Torsional resonance limits the velocity controller BW in most industrial drives. This situation is critical in high inertia applications, where the resonant frequency could be in the range of $10 \mathrm{~Hz}$ or less, affecting the command response of the drive. This situation is especially problematic in paper machine drives where a coordinated response of all section is required.

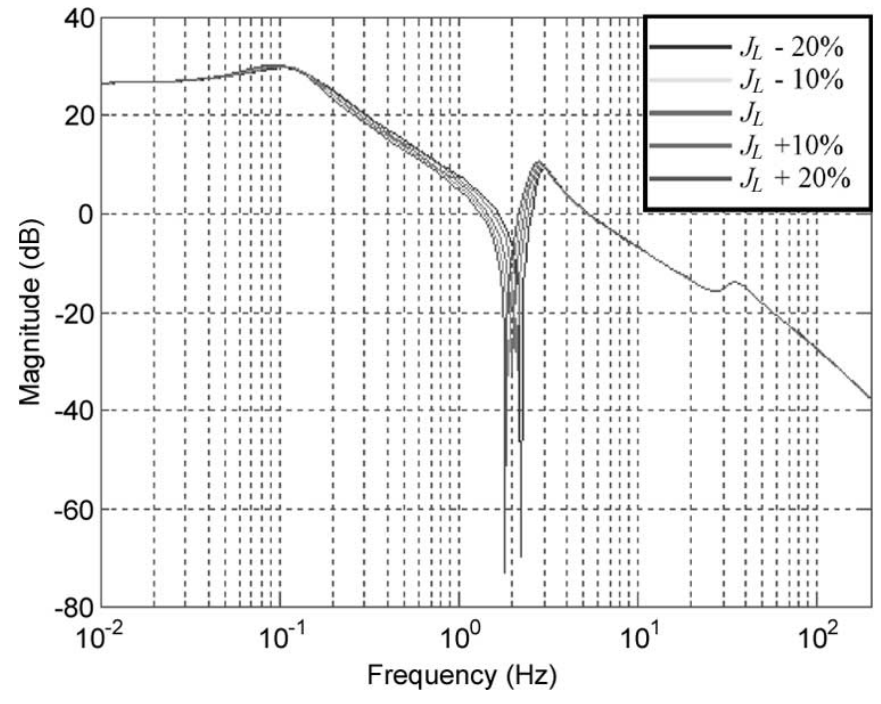

Fig. 16. Effect of variations of rolls inertia $J_{m}$ on the closed-loop frequency response when using observer-based acceleration feedback.

In this paper, two dynamic compensation techniques were evaluated. The first one is the use of a notch filter to cancel the resonant frequency. Based on the parameter variations investigated, the following conclusions can be drawn.

- Notch filters are effective in reducing torsional oscillation due to elastic shafts.

- Notch filters must be tuned to cancel the closed-loop resonant complex poles and fully attenuate the high magnitude at resonant frequency.

- If correctly tuned, the magnitude of oscillation is greatly reduced and transient oscillations vanish rapidly.

- Notch filter compensation is robust in the presence of $\pm 20 \%$ parameter variations.

The second compensation technique evaluated is acceleration feedback implemented using a rigid-body Luenberger observer. Based on the parameter variations investigated, the following conclusions can be drawn.

- Acceleration feedback is effective in reducing torsional oscillation due to elastic shafts. The magnitude at resonant frequency can be substantially reduced and the resonant frequency can be moved to a lower frequency inside the velocity controller BW.

- During a step command, the current response oscillates to damp out oscillation in the motor and roll velocities. Current peaks are increased, although values should be within the capability of the drives. In some cases, it may prove necessary to consider this issue in sizing the drive.

- Acceleration feedback compensation is robust in the presence of $\pm 20 \%$ parameter variations.

In conclusion, the use of either compensation technique can be recommended for the high inertia sections of paper machine, such as the Dryer section and the Calender. If implemented, the command response of velocity loops could be set for medium size inertia sections, improving the coordinated motion of the whole paper machine while reducing or eliminating sheet breaks due to transient loss of synchronization. 


\section{REFERENCES}

[1] M. A. Valenzuela, J. M. Bentley, and R. D. Lorenz, "Evaluation of torsional oscillations in paper machines sections," in Proc. IEEE Industry Applications Society (IAS) Pulp and Paper Industry Tech. Conf., Victoria, Canada, Jun. 27/Jul. 1, 2004, pp. 15-22.

[2] R. Dhaouadi, K. Kubo, and M. Tobise, "Analysis and compensation of speed drive systems with torsional loads," IEEE Trans. Ind. Appl., vol. 30, no. 3, pp. 760-766, May/Jun. 1994.

[3] S. N. Vukosavic and M. R. Stojic, "Suppression of torsional oscillations in a high-performance speed servo drive," IEEE Trans. Ind. Electron., vol. 45, no. 1, pp. 108-117, Feb. 1998.

[4] P. Schmidt and T. Rehm, "Notch filter tuning for resonant reduction in dual inertia systems," in Proc. IEEE Industry Applications Society Annu. Meeting, Phoenix, AZ, Oct. 1999, pp. 1730-1734.

[5] G. Ellis and R. D. Lorenz, "Resonant load control methods for industrial servo drives," in Proc. IEEE Industry Applications Society (IAS) Annu. Meeting, Rome, Italy, Oct. 2000, pp. 1438-1445.

[6] G. Younkin, "Compensating structural dynamics for servo driven industrial machines with acceleration feedback," in Proc. IEEE Industry Applications Society (IAS) Аnпu. Meeting, Seattle, WA, Oct. 2004, pp. 1881-1890.

[7] P. B. Schmidt and R. D. Lorenz, "Design principles and implementation of acceleration feedback to improve performance of DC drives," IEEE Trans. Ind. Appl., vol. 28, no. 3, pp. 594-599, May/Jun. 1992.

[8] R. D. Lorenz, T. A. Lipo, and D. W. Novotny, "Motion control with induction motors," Proc. IEEE (Special Issue on Power Electronics and Motion Control), vol. 82, no. 8, pp. 1215-1240, Aug. 1994.

[9] R. D. Lorenz and K. Van Patten, "High resolution velocity estimation for all digital, AC servo drives," IEEE Trans. Ind. Appl., vol. 27, no. 4, pp. 701705, Jul./Aug. 1991.

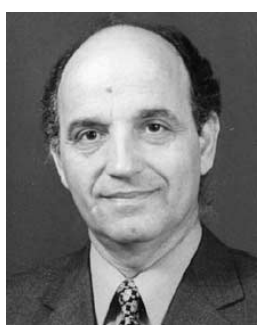

M. Aníbal Valenzuela (A'88-SM'01) was born in Santiago, Chile. He received the B.S. and M.S. degrees in electrical engineering from the University of Chile, Santiago, Chile, in 1976 and 1978, respectively.

Since 1978, he has been an Associate Professor at the Department of Electrical Engineering, University of Concepción, Concepción, Chile, where he is involve in the area of electric machines and drives. From August 1998 to June 1999, he spent his sabbatical leave at the Wisconsin Electric Machines and Power Electronics Consortium (WEMPEC), University of Wisconsin, Madison. He has several years of consulting activity in pulp and paper and in mining industries. His current research interests include motion control of industrial drives and coordinated motion of multi-axis systems, sensorless control of ac drives, and thermal evaluation of induction motors.

Mr. Valenzuela received the First Prize Paper Award for the best paper published in the IEEE TRANSACTIONS ON INDUSTRY APPLICATIONS in 2003.

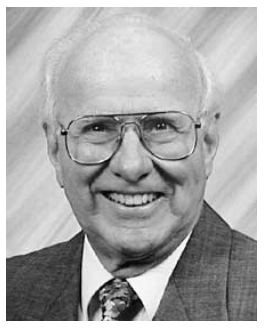

John Martin Bentley (M'54-SM'83-LS'94LF'99) received the B.S. degree in electrical engineering from the Pennsylvania State University, University Park, in 1951.

From 1951 to 1984 , he was with Westinghouse Electric Corporation, Pittsburgh, PA, and Buffalo, NY. He was awarded the company's high engineering position of Fellow and became recognized as an authority in the field of automatic control and drive systems for the paper industry worldwide, including the first digital sectional and winder drives in North America. From 1984 to 1991, he was a Principal Engineer at AllenBradley/Stromberg, Milwaukee, WI, where he was responsible for in-depth technical and marketing guidance to this multinational joint venture. During this period, he was instrumental in introducing the ac vector drive system to the North American market. Over 1000 sections, totaling approximately $200000 \mathrm{hp}$, were applied on sectional, winder, and finishing equipment drives, including the first sectional ac paper machine drive in North America. From 1991 until his retirement in 1996, he was a Principal Engineer at ABB Industrial Systems Inc., New Berlin, WI, and ABB Industry OY, Helsinki, Finland. He provided application and design guidance to the worldwide ABB Pulp and Paper Systems Drives organization, primarily in the area of high-performance ac drive and control systems. He had also been an author and instructor in his areas of expertise for many years. Since his 1996 retirement from ABB, he has continued to consult on a part-time basis, providing expertise in the areas of electromechanical influences on high-performance drive systems, cable and grounding techniques for controlling ac drive system induced common mode currents, and general guidance for the selection and application of highperformance ac drive systems.

Mr. Bentley was appointed to the status of Technical Association of the Pulp and Paper Industry (TAPPI) Fellow in 1986 and was elected as an IEEE Fellow "For contributions to the application and design of electrical drive systems in the pulp and paper industry" in 1999. The Fellow status is one of the most prestigious honors bestowed by TAPPI and IEEE, and he is the only living member that has been awarded the Fellow status by both organizations. He was acknowledged by the IEEE Industry Applications Society as he was a recipient of the Pulp and Paper Industry Committee Meritorious Achievement Award in 1979. He was recognized for his professional contribution within the TAPPI through the TAPPI Engineering Division Award and the E. H. Neese Prize in 1981.

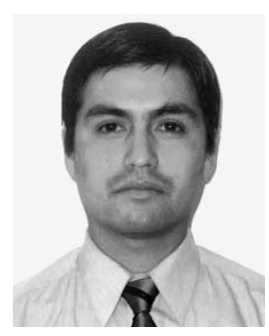

Andrés Villablanca was born in Concepción, Chile, on 1978. He received the electrical engineering degree from the University of Concepción, Concepción, Chile, in 2004.

His research interests include high-performance $\mathrm{dc}$ and ac drives and control of drives and servo systems.

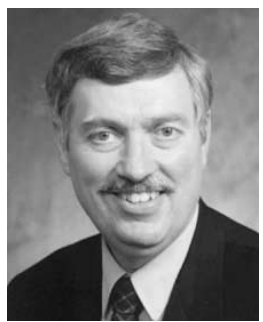

Robert D. Lorenz (S'83-M'84-SM'91-F'98) received the B.S., M.S., and Ph.D. degrees from the University of Wisconsin, Madison, in 1969, 1970, and 1984, respectively and the M.B.A. degree from the University of Rochester, Rochester, NY, in 1980.

From 1972 to 1982 , he was a Member of the Research Staff at Gleason Works, Rochester, NY, working principally on high-performance drives and synchronized motion control. Since 1984, he has been a Member of the Faculty of the University of Wisconsin, Madison, where he is the Mead Witter Foundation Consolidated Papers Professor of Controls Engineering at the Departments of Mechanical Engineering and Electrical and Computer Engineering. He is the Co-Director of the Wisconsin Electric Machines and Power Electronics Consortium, which will celebrate its 25th anniversary in 2006. It is the largest industrial research consortium on motor drives and power electronics in the world. He was a Visiting Research Professor at the Electrical Drives Group, Catholic University of Leuven, Leuven, Belgium, in the Summer of 1989, and in the Power Electronics and Electrical Drives Institute, Technical University of Aachen, Aachen, Germany, in the summers of 1987, 1991, 1995, 1997, and 1999, and was the SEW Eurodrive Guest Professor from September 1, 2000 to July 7, 2001. He is also the thrust leader for control and sensor integration at the Center for Power Electronic Systems, a National Science Foundation (NSF) Engineering Research Center that is a joint ERC with the Virginia Polytechnic Institute, Rensselaer Polytechnic Institute, University of Puerto Rico-Mayaguez, and North Carolina A\&T. His current research interests include sensorless electromagnetic motor/actuator technologies, realtime signal processing and estimation techniques, precision multi-axis motion control, and ac/dc drive and high precision machine control technologies. He has authored more than 170 published technical papers and is the holder of 23 patents with three more pending.

Dr. Lorenz is the IEEE Division II Director from 2005 to 2006, and was the IEEE Industry Applications Society (IAS) President in 2001, a Distinguished Lecturer of the IEEE IAS for 2000-2001, past Chair of the IAS Awards Department, past Chairman of the IAS Industrial Drives Committee, and is a Member of the IAS Industrial Drives Committee, Electrical Machines Committee, Industrial Power Converter Committee, and Industrial Automation and Control Committee. He is the past Chair of the Periodical Committee and current Chair of the Periodicals Review Committee for the IEEE Technical Activities Board. $\mathrm{He}$ is a Member of the IEEE Sensor Council AdCom. He is also a Member of the American Society of Mechanical Engineers (ASME), Instrument Society of America (ISA), and The International Society for Optical Engineers (SPIE). He is a Registered Professional Engineer in the states of New York and Wisconsin. He was awarded the 2003 IEEE IAS Outstanding Achievement Award, which honors his outstanding contributions and technological developments in the application of electricity to industry. He has won 18 prize paper awards. 